Publ. RIMS, Kyoto Univ.

12 Suppl. (1977), 53-68.

\title{
Sharp Fronts of Paired Oscillatory Integrals
}

\author{
by \\ Lars GÅRDING*
}

\section{Introduction}

Extending the work of Lax [6], using the theory of oscillatory integrals and Fourier integral operators, Duistermaat and Hörmander [3] constructed global parametrices of fundamental solutions of strongly hyperbolic differential operators with smooth coefficients. The singular spectra of these objects are certain Lagrangean manifolds. The explicit formulas make it possible at least in principle to see how such a parametrix behaves close to its singular support. In particular, it should be possible to investigate the existence and non-existence of sharp fronts. These are defined as follows. Let $Y$ be a closed subset of a manifold $X$ containing the singular support of a distribution $F$ and let $U$ be a component of $X \backslash Y$ whose boundary conatins a point $x_{0}$ of $Y$. We say that $F$ is sharp or has a sharp front at $x_{0}$ from $U$ if there is a neighborhood $V$ of $x_{0}$ such that $F$ has a $C^{\infty}$ extension from $U$ to $\bar{U} \cap V$. When this does not happen, $F$ is said to be diffuse or to have a diffuse front at $x_{0}$ from $U$. For instance, the distributions in one variable $(x \pm i 0)^{-1}$ are diffuse from both sides of the origin but their difference $2 \pi i \delta(x)=(x-i 0)^{-1}$ $-(x+i 0)^{-1}$ is sharp from both sides. The singular support of the forward fundamental solution of a second order hyperbolic differential operator is the corresponding forward light-cone. The fundamental solution is sharp from the outside for the trivial reason that it vanishes outside the cone. On the other hand, the classical parametrix construction by Hadamard shows that it is diffuse and sharp from inside the cone according as the number $n$ of variables is odd or even. In the latter case, when $n>2$ and the operator is homogeneous with constant coefficients, the inside of the light-cone is a lacuna, i.e. the fundamental solution vanishes there.

\footnotetext{
Recived August 30, 1976.

* Lunds University.
} 
As it turns out, oscillatory integrals as defined in [4] hardly ever define distributions with sharp fronts. But in parametrices of, e.g., forward fundamental solutions of hyperbolic operators these integrals occur in pairs whose sums may have sharp fronts. The pairing involved is actually an intrinsic affair connecting oscillating integrals with phase functions of opposite signs in such a way that the pairing survives pssages to equivalent phase functions, the only ambiguity being a change of sign which can be interpreted as the square of the Maslov index.

Continuing work by Petrovsky [7], the paper [1] by Atiyah-BottGårding dealt with sharp fronts and lacunas of forward fundamental solutions of hyperbolic operators with constant coefficients. The main tool was a topological criterion for the existence of lacunas invented by Petrovsky. A local version of it, the local Petrovsky criterion, is tied to the existence of sharp fronts. We shall extend it to distributions defined by paired oscillatory integrals and apply the results to fundamental solutions of strongly hyperbolic differential operators with variable coefficients. Our results are far from complete. The criterion in its general form remains a conjecture and its invariance and stability properties have to be made precise, but already in simple cases it yields some new results.

\section{$\S 1$. Paired oscillatory integrals.}

I shall use the notations and results of Hörmander [4] about oscillatory integrals. Let $X$ be a $C^{\infty}$ manifold of dimension $n$ and $S^{m}\left(X, \mathbb{R}^{N}\right)$ the class of complex $C^{\infty}$ functions from $X \times \boldsymbol{R}^{N}$, called amplitude functions, such that, for all $\alpha$ and $\beta$,

$$
\left|D_{x}{ }^{\alpha} D_{\theta}{ }^{\beta} a(x, \theta)\right|=O\left(\left(1+\mid \theta_{1}^{1}\right)^{m-\beta}\right)
$$

locally uniformly in $x$. Such an amplitude function $a(x, \theta)$ is said to be regular if it has an asymptotic expansion

$$
a(x, \theta) \sim \sum_{p=0}^{\infty} a_{m-p}(x, \theta)
$$

where $a_{m-p} \in S^{m-p}\left(X, \boldsymbol{R}^{N}\right)$ is homogeneous of degree $m-p$ in $\theta$ for large values of $\theta$ and $a-a_{m}-\cdots-a_{m-p} \in S^{m-p-1}\left(X, \boldsymbol{R}^{N}\right)$ for every $p$. In particular, all terms $a_{m-p}$ are uniquely determined by $a$ for all sufficiently large values of $\theta$. 
A regular phase function is a real $C^{\infty}$ function $\varphi(x, \theta)$ from $X \times\left(\boldsymbol{R}^{V} \backslash 0\right)$, homogeneous of degree 1 in $\theta$ such that the system $d \varphi_{\theta}(x, \theta)$ has rank $N$ when $\varphi_{\theta}(x, \theta)=0$. Restricted to $\varphi_{\theta}=0$, the $\operatorname{map}(x, \theta) \rightarrow\left(x, \varphi_{x}\right)$ then defines a corresponding Lagrangean manifold $\Lambda$ of dimension $n$ in $T^{*}(X) \backslash 0$, the cotangent bundle of $X$ minus its zero section. This manifold contains the singular spectrum of the oscillatory integral with any amplitude function $a(x, \theta)$

$$
I(a, \varphi, x)=\int a(x, \theta) e^{i \varphi(x, \theta)} d \theta .
$$

Two regular phase functions, $\varphi(x, \theta)$ from $X \times\left(\mathbb{R}^{N} \backslash 0\right)$ and $\widetilde{\varphi}(x, \tilde{\theta})$ from $X \times\left(\boldsymbol{R}^{\tilde{N}} \backslash 0\right)$, are said to be equivalent near a point $\left(x_{0}, \xi_{0}\right)$ of $T^{*}(X) \backslash 0$ if they generate the same Lagrangean manifold near this point, i.e. if there is a diffeomorphism $(x, \theta) \rightarrow(x, \tilde{\theta})$ from an open neighborhood of $\left(x_{0}, \theta_{0}\right)$ on $\varphi_{\theta}=0$ to an open neighborhood of $\left(x_{0}, \tilde{\theta}_{0}\right)$ on $\widetilde{\varphi}_{\tilde{\theta}}=0$ such that $\varphi_{x}(x, \theta)=\widetilde{\varphi}_{x}(x, \tilde{\theta})$, both sides reducing to $\xi_{0}$ when $x=x_{0}, \theta=\theta_{0}$, $\tilde{\theta}=\tilde{\theta}_{0}$. Under these circumstances, Theorem 3.1.4 and 3.2.1 of [4] show that

$$
N-\widetilde{N}-\operatorname{rank} \varphi_{\theta \theta}-\operatorname{rank} \widetilde{\varphi}_{\tilde{\theta} \tilde{\theta}}=0
$$

on $\Lambda$ and that the difference

$$
\sigma=\operatorname{sgn} \varphi_{\theta \theta}-\operatorname{sgn} \widetilde{\varphi}_{\bar{\theta} \bar{\theta}}
$$

is constant on $\Lambda$ close to $\left(x_{0}, \xi_{0}\right)$. Here $\operatorname{sgn}=\operatorname{sgn}_{+}-\operatorname{sgn}$ - and rank $=\operatorname{sgn}_{+}$

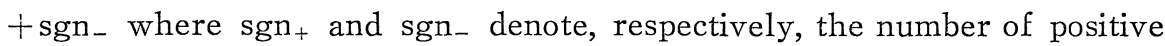
and negative eigenvalues of a matrix. Further, given a regulan amplitude function $a$ in $S^{-\mu-N / 2}\left(X, \mathbb{R}^{N}\right)$ with support in a sufficiently small conical neighborhood of $\left(x_{0}, \theta_{0}\right)$, there is another one, $\widetilde{a}$ in $S^{-\mu-\tilde{N} / 2}\left(X, \mathbb{R}^{N}\right)$ with support in a conical neighborhood of $\left(x_{0}, \tilde{\theta}_{0}\right)$ such that, close to $x_{0}$,

$$
I(a, \varphi, x) \sim I(\widetilde{a}, \widetilde{\varphi}, x)
$$

modulo smooth functions. Finally, given a regular phase function $\varphi(x, \theta)$ and a point $\left(x_{0}, \theta_{0}\right)$ on $\varphi_{\theta}=0$, there is an equivalent phase function $\widetilde{\varphi}$ such that $\widetilde{\varphi}_{\bar{\theta} \tilde{\theta}}=0$ at the corresponding point $\left(x_{0}, \tilde{\theta}_{0}\right)$.

Changing the the sign of the phase function we pass from the Lagrangean manifold $\Lambda$ to the opposite manifold $-\Lambda$ obtained from $\Lambda$ by the reflection $(x, \xi) \rightarrow(x,-\xi)$. We shall see what happens to (4) under this 
process. Let us write the expansions of $a$ and $\widetilde{a}$ as

$$
a \sim \sum_{p=0}^{\infty} a_{-q-N} i^{q}, \quad \widetilde{a} \sum_{p=0}^{\infty} \widetilde{a}_{-\widetilde{q}-\tilde{N}} i^{\widetilde{q}} .
$$

where $q=-N / 2+p, \widetilde{q}=-\widetilde{N} / 2+p$ and the powers of $i$ turn out to be convenient later. Let us define companions to these regular amplitude functions as follows

$$
a^{\prime} \sim \sum_{p=0}^{\infty} a_{-q-N} i^{-q}, a^{\prime} \sim \sum_{p=0}^{\infty} \widetilde{a}_{-\tilde{q}-\tilde{N}^{-}} i^{-\widetilde{q}}
$$

Then, passing to companions and changing the sign of the phase function leaves the equivalence (4) unchanged apart from a factor \pm 1 . In fact, we have

Lemma. It follows from (4) that

$$
I\left(a^{\prime},-\varphi, x\right) \sim(-1)^{\sigma-} I\left(\widetilde{a}^{\prime},-\widetilde{\varphi}, x\right)
$$

modulo smooth functions where

$$
\sigma_{-}=\operatorname{sgn}_{-} \varphi_{\theta \theta}-\operatorname{sgn}_{-} \widetilde{\varphi}_{\tilde{\theta} \tilde{\theta}}
$$

is constant on $\Lambda$ close to $\left(x_{0}, \xi_{0}\right)$.

The proof is easily extracted from [4], the essential point being that the coefficients $c_{\alpha}=c_{\alpha}(A)$ of the formula (3.2.6) of [4] have the property that $c_{\alpha}(-A)=(-1)^{|\alpha| / 2} c_{\alpha}(A)$. It follows from the formula (3.2.11) of [4] that (7) holds with the factor $i^{N-\widetilde{N}-\sigma}$ on the right which, by virtue of (2) and (3) equals $(-1)^{\sigma_{-}}$.

Note. It is easy to verify that (7), taken as a requirement that the two sides be proportional, uniquely determines the pairing $a_{-}>a^{\prime}$ up to a factor and that (7) then holds as written. Note that $(-1)^{\sigma_{-}}$is the square of $i^{\sigma_{-}}$, a factor which for oscillatory integrals accounts for the Maslov index ([4]pp. 154-163).

Oscillatory integrals of the type (1) will be called simple while those of the form

$$
I^{\varepsilon}(a, \varphi, x)=I(a, \varphi, x)+\varepsilon I\left(a^{\prime},-\varphi, x\right), \varepsilon= \pm 1,
$$


are said to be paired. Note that with assumptions as in the lemma, i.e. if $I(a, \varphi, x) \sim I(\widetilde{a}, \widetilde{\varphi}, x)$, then

$$
I^{\varepsilon}(a, \varphi, x) \sim I^{\tilde{\varepsilon}}(\widetilde{a}, \widetilde{\varphi}, x) \quad \text { where } \quad \widetilde{\varepsilon}=(-1)^{\sigma_{-}} \varepsilon .
$$

Polar coordinates. Let us write (1) in terms of the expansions (5) and (6),

$$
\begin{aligned}
& I(a, \varphi, x) \sim \sum_{p=0}^{\infty} \int a_{-q-N}(x, \theta) i^{q} e^{i \varphi(x, \theta)} d \theta \\
& I\left(a^{\prime},-\varphi, x\right) \sim \sum_{p=0}^{\infty} \int a_{-q-N}(x, \theta) i^{-q} e^{-i \varphi(x, \theta)} d \theta
\end{aligned}
$$

where $q=\mu-N / 2+p$. We shall introduce polar coordinates on the right sides using a real $C^{\infty}$ radius function $\gamma(\theta)>0$ from $\mathbb{R}^{N} \backslash 0$ of homogeneity 1 . Assuming that the amplitude functions are homogeneous when $r \geqq$, the result is

$$
\begin{aligned}
& I(a, \varphi, x) \sim \sum_{p=0}^{\infty} \int_{\tau=1} a_{-q-N}(x, \theta) \chi_{q}(\varphi(x, \theta)+i 0) \omega(\theta) \\
& I\left(a^{\prime},-\varphi, x\right) \sim \sum_{p=0}^{\infty} \int_{\lambda=1} a_{-q-N}(x, \theta) \chi_{q}(\varphi(x, \theta)-i 0) \omega(\theta)
\end{aligned}
$$

where each term differs from the corresponding term of (10), (11) by a $C^{\infty}$ function,

$$
\omega(\theta)=\theta_{1} d \theta_{2} \wedge \cdots \wedge d \theta_{N}-\theta_{2} d \theta_{1} \wedge d \theta_{3} \cdots<d \theta_{N}+\cdots
$$

and the $\chi_{q}(t \pm i 0)=\lim \chi_{q}(t \pm i \varepsilon), \varepsilon>0$, are distributions in one variable, boundary values on the real axis of the analytic functions

$$
\chi_{s}(z)=\Gamma(-s) z^{s}, \quad \chi_{p}(z)=z^{p}\left(\log z^{-1}+c_{p}\right) / p .
$$

where $-\pi<\arg z<\pi, p \geqq 0$ is an integer and $s \neq 0,1,2, \cdots$ and $c_{0}=0$, $c_{p}=p^{-1}+c_{p-1}$. Putting

$$
\chi_{s}^{\varepsilon}(t)=\chi_{s}(t+i 0)+\varepsilon \chi_{s}(t-i 0)
$$

we can now write the paired oscillatory integral (9) as

$$
I^{\varepsilon}(a, \varphi, x) \sim \sum_{p=0}^{\infty} \int_{r=1} a_{-q-N}(x, \theta) \chi_{q}^{\varepsilon}(\varphi(x, \theta)) \omega(\theta) .
$$

Note that if $s$ is an integer, then $\chi_{s}^{\varepsilon}(t)$ is one of the successive integrals or derivatives of 


$$
\chi_{0}^{+}(t)=2 \log |t|^{-1}, \quad \chi_{0}^{-}(t)=-\pi i \operatorname{sgn} t
$$

and hence $\chi_{s}^{+}$is diffuse and $\chi_{s}^{-}$sharp from both sides of the origin. When $s$ is a half-integer meaning that $2 s$ is an integer but $s$ is not, then, putting $t_{\varepsilon}=\max (0, \varepsilon t)$,

$$
\chi_{s}^{\varepsilon}(t)=\text { const } t_{\varepsilon}^{s}
$$

is sharp at the origin from the side $\varepsilon t<0$ and diffuse from the other.

\section{$\S 2 . \quad F o r w a r d$ parametrices of strongly hyperbolic operators.}

Let $P(x, D)$ where $D=\partial / i \partial x$ is the imaginary gradient be a smooth differential operator of order $m$ from some open subset of $\mathbb{R}^{n}$ containing the origin and assume that $P$ is strongly hyperbolic with respect to the hyperplanes $x_{1}=$ const. We can then factor its principal symbol, suitably normalized, as $p_{1} \cdots p_{m}$ with real factors

$$
p_{k}(x, \xi)=\xi_{1}-c_{k}\left(x, \xi_{2}, \cdots, \xi_{n}\right) .
$$

The functions $c_{k}$ are smooth and of homogeneity 1 in $\xi$ and all different unless $\xi_{2}=0, \cdots, \xi_{n}=0$. When $x$ is close to the origin they define $m$ regular phase functions $\varphi_{1}, \cdots, \varphi_{m}$ depending on $\theta=\left(\theta_{2}, \cdots, \theta_{2}\right)$ and satisfying $p_{k}\left(x, \varphi_{k x}\right)=0, \varphi_{k}=x_{2} \theta_{2}+\cdots+x_{n} \theta_{n}$ when $x_{1}=0$. There is also an involution $k \rightarrow k^{\prime}$ defined by $p_{k}(x,-\xi)=-p_{k^{\prime}}(x, \xi)$ so that also $\varphi_{k}(x,-\theta)$ $=-\varphi_{k^{\prime}}(x, \theta)$. Let $H(t)=(1+\operatorname{sgn} t) / 2$ be the Heaviside function. By Lax's construction [6], $P$ has a right forward parametrix $H\left(x_{1}\right) E(x)$ where $P E \sim 0$ and, for small $x$,

$$
E(x)=E_{1}(x)+\cdots+E_{m}(x)
$$

where

$$
E_{k}(x) \sim \int a_{k}(x, \theta) e^{i \varphi_{k}(x, \theta)} d \theta=I\left(a_{k}, \varphi_{k}, x\right)
$$

with regular amplitude functions $a_{k}$ in $S^{1-m}\left(X, \mathbb{R}^{n-1}\right), X$ being a neighborhood of zero in $\boldsymbol{R}^{n}$. Expanding $a_{k} \sim \sum_{p=1-m}^{\infty} b_{k p}$ where $b_{k p}$ is homogeneous of degree $p$ for large values of $\theta$, Lax's construction also shows that $b_{k^{\prime} p}=(-1)^{p} b_{k p}$ and this turns out to imply that $E_{k^{\prime}} \sim(-1)^{n-1} I\left(a_{k}{ }^{\prime},-\varphi_{k}, x\right)$. In other words, close to the origin, 


$$
E(x) \sim 2^{-1} \sum_{k} I^{\varepsilon}\left(a_{k}, \varphi_{k}, x\right), \varepsilon=(-1)^{n-1}
$$

is a sum of paired oscillatory integrals.

Lax's construction only holds close to the origin but Duistermaat and Hörmander [3] have shown that if $X$ is an open connected neighborhood of 0 which is pseudoconvex with respect to the bicharacteristics of $p_{1}, \cdots, p_{m}$ passing through the origin, then every $E_{k}$ has an extension to $X$, unique modulo smooth functions and denoted here by $E_{k}$, such that $E_{1}+\cdots+E_{m}$ multiplied by $H\left(x_{1}\right)$ is a forward right parametrix. Moreover, to every $\left(x_{0}, \xi_{0}\right)$ in the corresponding Lagrangean manifold $\Lambda_{k}$ there is a regular phase function $\varphi(x, \theta)$ representing $\Lambda_{k}$ at $\left(x_{0}, \xi_{0}\right)$ such that, close to $x_{0}$,

$$
E_{k}(x) \sim I(a, \varphi, x)
$$

for some regular amplitude function $a(x, \theta)$ in $S^{-\mu-N / 2}$ where $N=\operatorname{dim} \theta$ and $\mu=m-1-(n-1) / 2$. This also applies to $E_{k}$, with the phase function $-\psi(x, \theta)$ corresponding to the Lagrangean manifold $-\Lambda_{k}$. The lemma applied to a chain of overlapping neighborhoods shows that

$$
E_{k}(x)+E_{k}(x) \sim I^{\varepsilon}(a, \varphi, x)
$$

for some $\varepsilon= \pm 1$. Passing to an equivalent phase function changes the right side according to (1.9). The following theorem sums up the situation.

Theorem 1. Outside the origin, the forward right parametrix $E(x)$ of a strongly hyperbolic differential operator $P(x, D)$ of order $m$ is a linear combination of paired oscillatory integrals having asymptotic expansions

$$
\sim \sum_{p=1}^{\infty} \int_{r=0} b_{p}(x, \theta) \chi_{y+p}^{\varepsilon}((x, \theta)) \omega(\theta)
$$

with $C^{\infty}$ amplitudes $b_{p}$. Here $\nu=m-1-(n-1-N) / 2, N=\operatorname{dim} \theta$ and $\varphi$ is a regular phase function belonging to the Lagrangean manifold of $P$ issuing from the origin and $\varepsilon=1$ or -1 . Changing to an equivalent phase function $\widetilde{\varphi}$ multiplies $\varepsilon$ by the factor $(-1)^{\sigma_{-}}$where

$$
\sigma_{-}=\operatorname{sgn}_{-} \varphi_{\theta \theta}-\operatorname{sgn}_{-} \widetilde{\varphi}_{\bar{\theta} \bar{\theta}}
$$


is locally constant on the Lagrangean manigold. Close to the origin $\varphi$ can be chosen so that $N=n-1, \varphi=x_{2} \theta_{2}+\cdots+x_{n} \theta_{n}$ when $x_{1}=0$ and then $\varepsilon=(-1)^{n-1}$.

\section{§ 3. Sharpness of paired oscillatory integrals. The Petrovsky criterion.}

Consider a paired oscillatory integral

$$
G(x) \sim \sum_{p=0}^{\infty} \int_{r=1} b_{-\mu-N / 2-p}(x, \theta) \chi_{-\mu-N / 2+p}^{\varepsilon}(\varphi(x, \theta)) \omega(\theta)
$$

and assume that all the amplitude functions vanish outside some conical neighborhood of a point $\left(x_{0}, \theta_{0}\right)$ where $\varphi_{\theta}=0$. As remarked before, we may assume that $\operatorname{rank} \varphi_{\theta \theta}=0$, i.e. corank $\varphi_{\theta \theta}=N$ at this point. When $N=1$, then the right side of (1) is a sum of distributions

$$
x \rightarrow \chi_{-k-1 / 2+p}^{\varepsilon}(\varphi(x, \theta)), \quad \gamma(\theta)=1,
$$

independent of $\theta$, multiplied by regular functions. Also, close to $x_{0}$, the projection $Y=\pi \Lambda$ on $x$-space of $\Lambda$ is a regular hypersurface whose equation is $\varphi(x, \theta)=0$ (note that $\varphi_{x} \neq 0$ ). Hence, if $\mu$ is an integer, $G(x)$ is sharp from one side of $Y$ and non-sharp from the other and, if $\mu$ is a half-integer, $G(x)$ is either diffuse $(\varepsilon=1)$ or sharp $(\varepsilon=-1)$ from both sides. For fundamental solutions of hyperbolic differential operators with constant coefficients, this result is due to Davidova (1946) (quoted by Borovikov [2]).
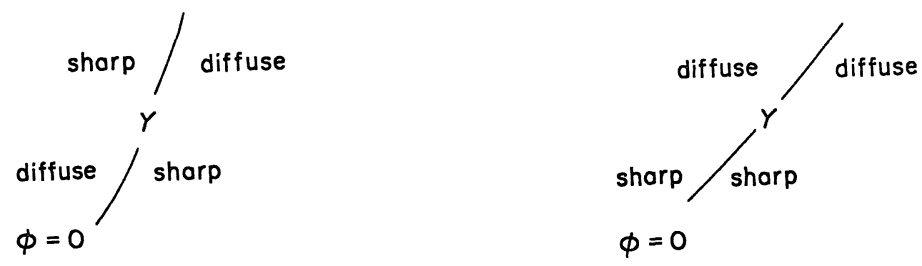

Figure 1. Behavior of paired oscillatory integrals when corank $\varphi_{\theta \theta}=1$. Note that for simple oscillatory integrals with $\chi(t+i \varepsilon 0)$ instead of $\chi^{\varepsilon}(t)$, none of the distributions $x \rightarrow \chi_{\mu-1 / 2+p}(\varphi)$ is sharp from either side of $Y=\pi \Lambda$.

When the corank exceeds $1, Y$ ceases to be a manifold and the behavior of paired oscillatory integrals is more complicated. For hyperbolic operators with constant coefficients there is a topological criterion 
for sharpness, the local Petrovsky criterion, ([1] Ch III) covering such cases. We shall extend it to paired oscillatory integrals

$$
F^{\varepsilon}(x)=\int b(x, \theta) \chi_{q}^{\varepsilon}(\varphi(x, \theta)) d \theta
$$

where $q$ is an integer or a half-integer, $x$ belongs to a $C^{\infty}$ manifold $X$, $\theta=\left(\theta_{1}, \cdots, \theta_{N-1}\right)$ are inhomogeneous coordinates on $\gamma=1$ and $b(x, \theta)$ and $\varphi(x, \theta)$ are the restrictions of the homogeneous functions $b(x, \theta)$ and $\varphi(x, \theta)$ to $\gamma=1$. The amplitude function $b$ is supposed to have compact support in the $\theta$ variables and $\varphi$ is supposed to be a regular phase function. The associated Lagrangean manifold $\Lambda$ then consists of all points $\left(x, s \varphi_{x}\right)$ such that $\varphi=\varphi_{\theta}=0$ and $s>0$. We let $Y=\pi \Lambda$ be its projection on $X$. An almost analytic extension of a $C^{\infty}$ function $g(\theta)$ defined in an open set $O$ of $\boldsymbol{R}^{N-1}$ is a $C^{\infty}$ extension into an open set in $\mathbb{C}^{N-1}$, also denoted by $g$, such that $\partial g / \partial \bar{\theta}=0$ when $\theta$ is real. (See Hörmander [5]).

The idea is now to shift the integration in (2) from $\mathbb{R}^{N-1}$ into $\boldsymbol{C}^{N-1}$ using the formula

$$
\chi_{q}^{\varepsilon}(\varphi)=\chi_{q}(\varphi+i 0)+\varepsilon \chi_{q}(\varphi-i 0)
$$

and almost analytic extensions of the amplitude function $b$ and the phase function $\varphi$ to an open subset $\Omega$ of $\mathbb{C}^{i N-1}$. This will be done using certain chains and cycles. The zero set of $\varphi$ in $\Omega$ will be denoted by $\phi(x)$.

Petrovsky chains and cycles.

1. Integral $q$. Let $U$ be an open part of $X \times \operatorname{Re} \Omega$. Consider $C^{\infty}$ maps $v(x, \theta, t)$ into $\Omega$ from $U \times(t ; 0 \leqq t \leqq 1)$ such that

(i) $t=0 \Rightarrow v=\theta$

(ii) $t=0, \varphi(x, \theta)=0 \Rightarrow \operatorname{Im} \varphi_{\theta} v_{t}>0$

(iii) $t>0 \Rightarrow \varphi(x, v) \neq 0$.

Every point $\left(x_{0}, \theta_{0}\right)$ where $\varphi$ or $\varphi_{\theta} \neq 0$ has a neighborhood where such maps exist and we may even take $v$ independent of $x$ when $U$ is small enough. Next, let $c(x)$ be the chain $(\theta, t) \rightarrow v(x, \theta, t)$ where $0<t \leqq 1$, oriented by $d \theta d t>0$.

Definition. A Petrovsky chain for integral $q$ is 


$$
A(x, q, \varepsilon)=c(x)+\varepsilon \overline{c(x)} \subset \Omega \backslash \phi(x) .
$$

A Petrovsky cycle is

$$
\alpha(x, q, \varepsilon)=\partial A(x, q, \varepsilon) \subset \Omega \backslash \phi(x) .
$$

2. Half-integral $q$. Replace $\Omega$ by a two-sheeted cover $\Omega^{1 / 2}$ consisting of all $\left(x, \theta^{\prime}\right)=\left(x, \theta, \theta_{N}\right)$ with $(x, \theta)$ in $X \times \Omega$ and $\theta_{N}{ }^{2}-\varepsilon \varphi(x, \theta)=0$. Let $U$ be an open part of $X \times \operatorname{Re} \Omega$ and let $U^{1 / 2}$ be the corresponding two-sheeted cover. Consider $C^{\infty}$ maps $v=v\left(x, \theta^{\prime}, t\right)$ into $\Omega$ from $\operatorname{Re} U^{1 / 2}$ $\times(t ; 0 \leqq t \leqq 1)$ such that

(i) $t=0 \Rightarrow v=\theta$,

(ii) $t=0, \varepsilon \varphi(x, \theta)>0 \Rightarrow \operatorname{Im} \varepsilon \theta_{N} \varphi_{\theta} v_{t}>0$

(iii) $t>0 \Rightarrow \varepsilon \varphi(x, \theta)$ is not $>0$.

Every point $\left(x_{0}, \theta_{0}{ }^{\prime}\right)$ with $\varphi$ or $\varphi_{\theta} \neq 0$ has a neighborhood $U^{1 / 2}$ where such maps exist and we may even take $v$ independent of $x$ when $U$ is small enough. Next, let $c(x)$ be the chain $\left(\theta^{\prime}, t\right) \rightarrow v$ for $0<t \leqq 1$ oriented by $\theta_{N} d \theta d t>0$.

Definition. A Petrovsky chain for half-integral $q$ is

$$
A(x, q, \varepsilon)=c(x) \subset \Omega^{1 / 2} \backslash \phi(x)^{1 / 2} .
$$

A Petrovsky cycle is

$$
\alpha(x, q, \varepsilon)=\partial A(x, q, \varepsilon) \subset \Omega^{1 / 2} \backslash \phi(x)^{1 / 2} .
$$

Figure 2 below illustrates the simplest case $N=2$. The set $\phi(x)$ is then a number of real points and pairs of conjugate complex points in the complex $\vartheta$-plane.

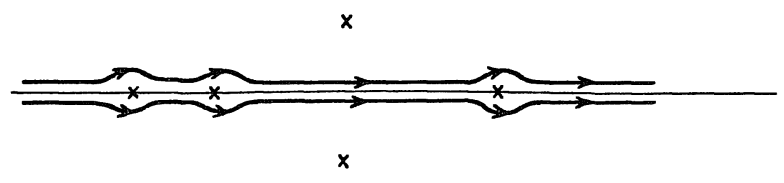

$q$ integer, $\varepsilon=1 \Rightarrow \alpha(x)=2 \operatorname{Re} \Omega$ detached from $\operatorname{Re} \phi(x)$.

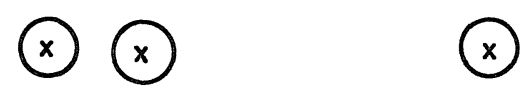

$q$ integer, $\varepsilon=-1 \Rightarrow \alpha(x)$ consists of loops around $\operatorname{Re} \phi(x)$ with alternate orientations. 

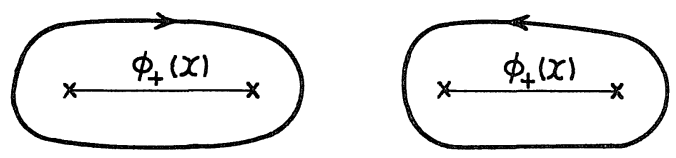

$q$ half-integer, $\varepsilon=1 \Rightarrow \alpha(x)$ projected down to $\Omega$ consists of loops around $\phi_{+}(x)$ : $\varphi(x, \theta) \geqq 0$ with alternate orientations.
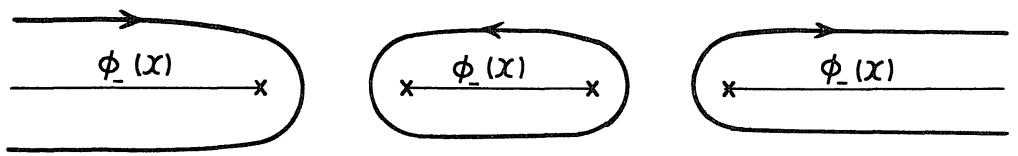

$q$ half-integer, $\varepsilon=-1 \Rightarrow \alpha(x)$ projected down to $\Omega$ consists of loops around $\phi_{-}(x)$ : $\varphi(x, \theta) \leqq 0$ with alternate orientations.

Figure 2. Petrovsky cycles when $N=2$.

Theorem 2. Let $O \subset \boldsymbol{R}^{N-1}$ be open, let $\varphi \in C^{\infty}(X \times 0)$ be a regular phase function with Lagrangean manifold $\Lambda$ and let $Y=\pi \Lambda$ be its projection on $X$. Let amplitude function $b(x, \theta) \in C^{\infty}(X \times 0)$ have compact support when restricted to compact parts of $X$. Let $b, \varphi$ be almost analytic extensions of $b, \varphi$ to an open subset $\Omega$ of $X \times \mathbb{C}^{N-1}$ preserving the support property of $b$. Then every point of $X \backslash Y$ has $a$ neighborhood $V$ such that Petrovsky chains $A(x, q, \varepsilon)$ and cycles $\alpha(x, q, \varepsilon)$ contained in $\Omega$ exist when $x \in V$, and, if $F^{\varepsilon}$ is given by (2), putting

$$
f(x, \theta)=b(x, \theta) \chi_{q}(\varphi(x, \theta)) d \theta
$$

we have

$$
F^{\varepsilon}(x)=\int_{\alpha(x)} f(x, \theta)-\int_{A(x)} d f(x, \theta) .
$$

Proof. Stokes' formula, the fact that $d f(x, \theta)$ vanishes of infinite order when $\theta$ is real and the properties of Petrovsky chains shows that the right side of (3) equals

$$
\int b(x, \theta)\left(\chi_{q}(\varphi(x, \theta)+i 0)+\varepsilon \chi_{q}(\varphi(x, \theta)-i 0)\right) d \theta
$$

with integration over $\boldsymbol{R}^{N-1}$. We shall now state a hypothesis, viz. 
The local Petrovsky criterion. Let $x_{0} \in Y$ and let $l$ be a smooth path in $X \backslash Y$ ending in $x_{0}$. There is a smooth $(N-1)$-cycle $\beta$ in $\Omega$ outside $\phi\left(x_{0}\right)$ such that, for $x \in l$ sufficiently close to $x_{0}, \beta$ is homologous to some Petrovsky cycle $\alpha(x, q, \varepsilon)$.

The following theorem, marked by a star, is partly a conjecture.

Theorem 3.* The local Petrovsky criterion implies that $F^{\varepsilon}(x)$ is sharp at $x_{0}$ from the component of $X \backslash Y$ containing $l$.

Sketch of a proof: By hypothesis, there is a $(n+1)$-chain $B(x)$ in $\Omega \backslash \phi(x)$ whose boundary consists of $\beta$ and a part of $\operatorname{Re} \Omega$. If $p<0$ is an integer, $f(x, \theta)$ is single-valued and we have

$$
F^{\varepsilon}(x)=\int_{\beta} f(x, \theta)-\int_{B(x)} d f(x, \theta)
$$

where $d f(x, \theta)$ vanishes of infinite order for real $\theta$. When $f(x, \theta)$ is analytic in the $\theta$ variables, this formula actually proves the theorem and there is a proof also for the other values of $p$. But in the non-analytic case, the choice of $B(x)$ as a function of $x$ presents a technical problem. In any case, the theorem is true when $N=2$ and $\phi\left(x_{0}\right)$ consists of a finite number of points. Figure 2 then shows immediately when a desired cycle exists and how to choose it. A simple description follows.

When $x$ is outside $Y$, the real zeros of the functions $\theta \rightarrow \varphi(x, \theta)$ are simple and hence the components of $\varphi \geqq 0$ and $\varphi \leqq 0$ are disjoint intervals. As $x$ tends to $x_{0} \in Y$ in a component of $X \backslash Y$, only two things may happen: adjoining intervals with $\varphi \neq 0$ collapse to a point or new real zeros of even multiplicity appear in the intervals that do not collapse. The fact of the matter is that $F^{\varepsilon}$ is sharp at $x_{0}$ from a component of $X \backslash Y$ if, as $x$ tends to $x_{0}$,

1) $q$ integer, $\varepsilon=1$, only collapses occur and no new zeros appear

2) $q$ integer, $\varepsilon=-1$, no collapses occur and only new zeros appear

3) $q$ half-integer, $\varepsilon=1$, new zeros appear only when $\varphi<0$ and all collapses involve just one component of $\phi_{+}(x)$

4) $q$ half-integer, $\varepsilon=-1$, new zeros appear only when $\varphi>0$ and all collapses involve just one component of $\phi_{-}(x)$. 
To prove e.g. 1), let $c$ be the real $\theta$-axis with small intervals around the collapses replaced by semi-circles in the upper half-plane, put $\beta=c+\bar{c}$ and let $B$ be the union of the resulting circles oriented by the sign of $\operatorname{Im} \theta$. Then

$$
F^{\varepsilon}(x)=\int_{\beta} f(x, \theta)-\int_{B} d f(x, \theta)
$$

where, since $d f(x, \theta)$ vanishes of infinite order when $\theta$ is real, the right side is a $C^{\infty}$ function of $x$ as long as no point of $\phi(x)$ enters $B$ or leaves the real axis. The proofs in the other cases are similar. If sharpness holds for all permitted amplitude functions, our four cases exhaust all possibilities.

Note. The cycle $\beta$ that appears in the Petrovsky condition can probably serve for all regular phase functions close to $\varphi$, making the criterion stable under small changes of $\varphi$. It remains to make this statement precise and also to prove that the criterion is invariant under shifts to equivalent phase functions. Applied to the parametrix $E(x)$ of the fundamental solution of a second order strongly hyperbolic differential operator in $n$ variables, the criterion shows that $E(x)$ is sharp and diffuse at the origin from inside the light-cone according as $n$ is even or odd, a result implicit already in the classical construction by Hadamard (Lectures in Cauchy's Problem...(1921), Dover Publ.) For higher order equations, corresponding results would imply sharpness at the origin from inside regions corresponding to lacunas in the constant coefficient case. Here the proofs will require a modified Petrovsky criterion involving the entire parametrix and not only each paired term separately.

Application to cusps and swallow's tails. Let $\varphi(x, \theta)$ with $\operatorname{dim}$ $\theta=1$ be a regular phase function with just one isolated singularity $\left(x_{0}\right.$, $\theta_{0}$ ) where $\varphi=\varphi_{\theta}=\varphi_{\theta \theta}=0$ but $\varphi_{\theta \theta \theta} \neq 0$. then there are $C^{\infty}$ variables $s=$ $s(x, \theta)$ and $y=y(x)$ such that $s\left(x_{0}, \theta_{0}\right)=0, y\left(x_{0}\right)=0$ and

$$
\varphi(x, \theta)=s^{3} / 3+y_{1} s+y_{2} .
$$

The curve $\varphi=0, \varphi_{s}=0$ in the $y_{1}, y_{2}$-plane has a cusp dividing the plane into two regions where $s_{-}>\varphi(x, \theta)$ has one or three real zeros. The 
points of Figure 3 show the positions of the zeros in the various parts and, when relevant, also the intervals of $\phi_{+}(x)$ and $\phi_{-}(x)$. According to the rules above, the paired oscillatory integral

$$
F^{\varepsilon}(x)=\int b(x, \theta) \chi_{q}^{\varepsilon}(\varphi) d \theta
$$

has sharp and diffuse fronts as given by Figure 3.
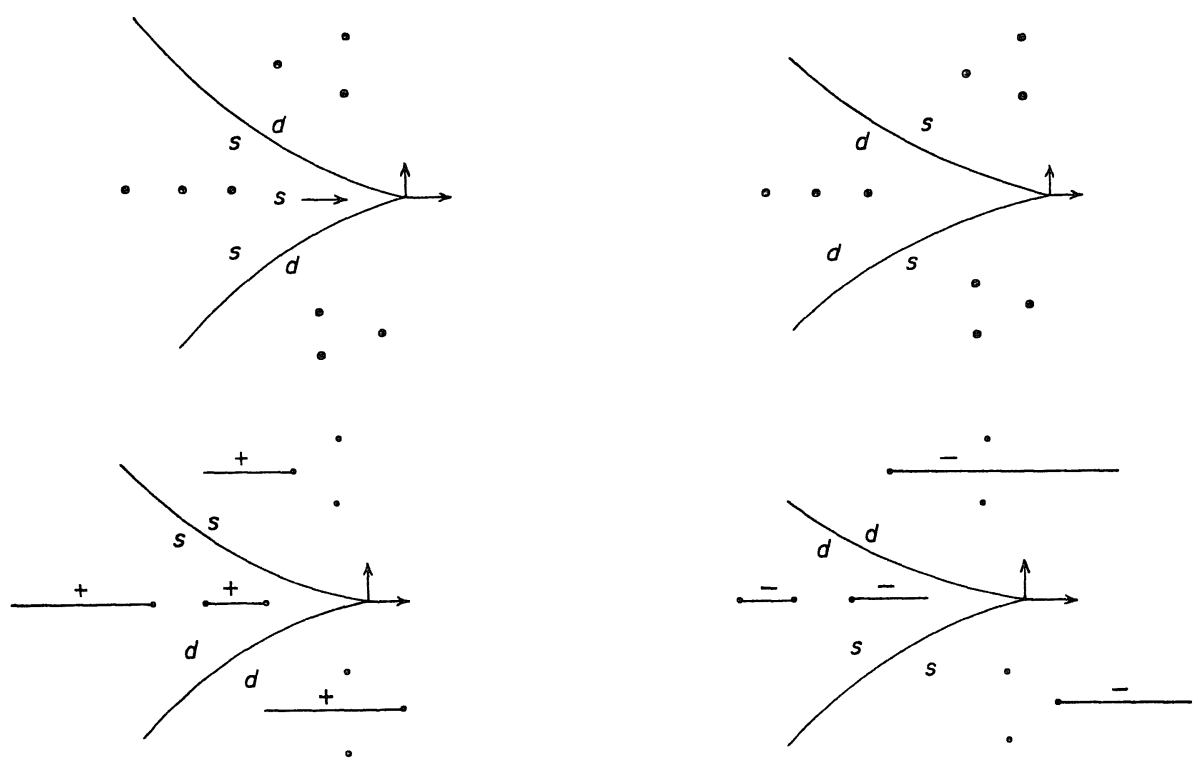

Figure 3. Sharp and diffuse fronts at cusps. The origin of the coordinates $y_{1}, y_{2}$ is at the vertex, the $y_{1}$-axis is vertical. There are four cases, $q=$ integer or half-integer, $\varepsilon= \pm 1$. The dots indicate the positsons of the zeros of the polynominal (4) in the complex plane, either all three real or else one real and a conjugate pair.

Next, assume that has an isolated singularity as before but that $\varphi^{(4)}(x, \vartheta)$ is the first non-vanishing derivative at the singular point. Then there are $C^{\infty}$ variables $s=s(x, \theta)$ and $y=y(x)$ such that, after eventually changing $\varphi$ to $-\varphi$,

$$
\varphi(x, \vartheta)=s^{4} / 4+y_{1} s^{2} / 2+y_{2} s+y_{3} .
$$

The surface $\varphi=\varphi_{s}=0$ in $y_{1}, y_{2}, y_{3}$-space is then a swallow's tail whose sections with the planes $y_{1}=$ const are sketched in Figure 4 together with the positions of the zeros of the polynomials (5). 

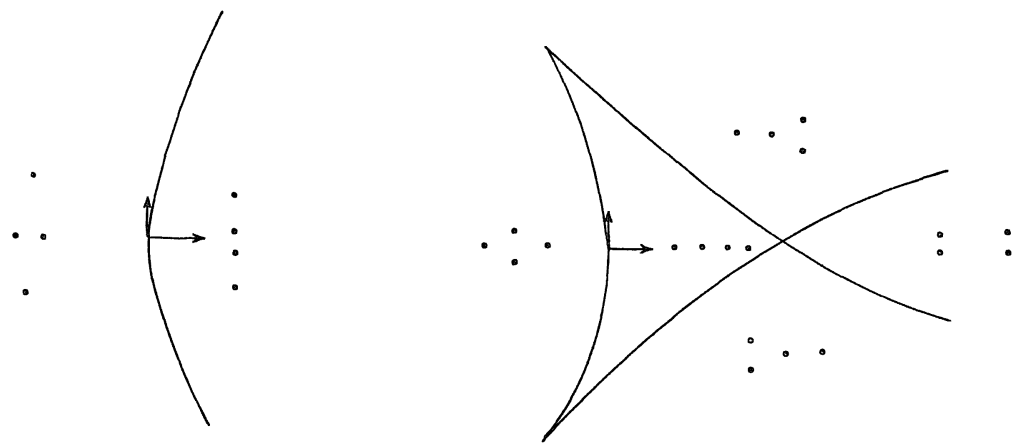

Figure 4. Positions of the zeros of (5) for various values of $y$. The arrows are at the origin of coordinates $y_{2}, y_{3}$. The $y_{2}$-axis is vertical.

Applying our criteria we get a Figure 5 to be interpreted as Figure 3. To its first column one might add that $F^{\varepsilon}$ is sharp at $y_{2}=0$ from inside the tail. In case a swallow's tail appears at the outer wave front of the forward fundamental solution of a second order hyperbolic differential operator in an odd (even) number of variables, the second (fourth) column applies. The question of sharpness in this situation was brought to my attention by J. J. Duistermaat.

$$
s / d
$$

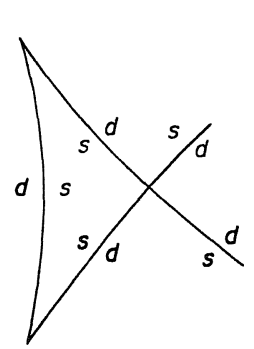

integer, +

$$
d / s
$$

$$
d / d
$$
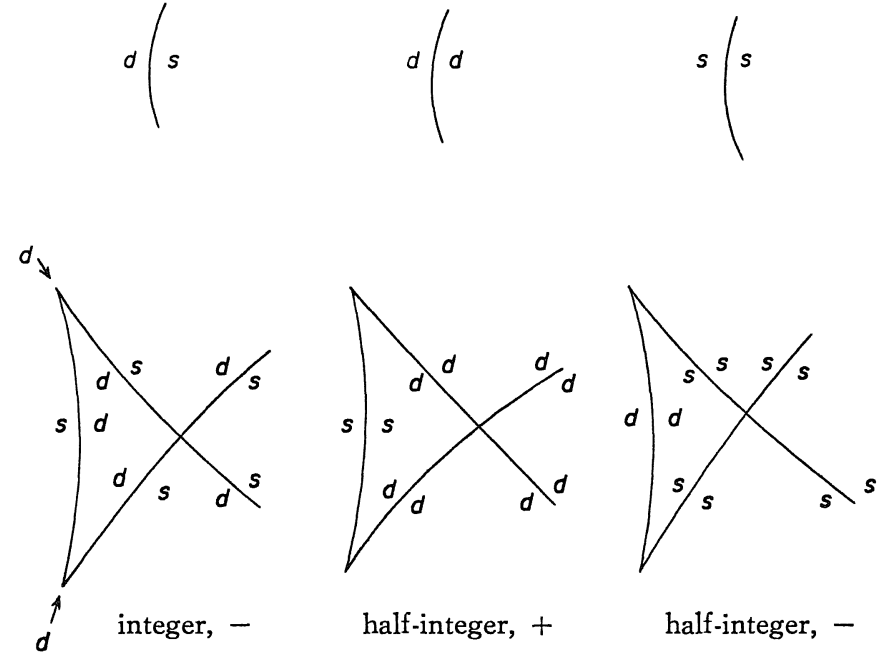

half-integer, +

\section{7}




\section{References}

[1] Atiyah M. F., Bott R. and Gårding L., Lacunas for hyperbolic differential operators with constant coefficients, Acta Math., 131 (1973), 145-206.

[2] Borovikov, V. A., Some sufficient conditions for the absence of lacunas, Mat. Sb., 55 (98) (1961), 237-247.

[3] Duistermaat, J. J. and Hörmander, L., Fourier integral operators II, Acta Math., 128 (1972), 183-269.

[4] Hörmander, L., Fourier integral operators I, Acta Math., 127 (1971), 79-183.

[5] Hörmander, L., Notes for the Nordic Summer School of Mathematics, 1969.

[6] Lax, P., Asymptotic solutions of oscillatory initial value problems, Duke Math. J., 24 (1957), 627-646.

[7] Petrovsky, I. G., On the diffusion of waves and the lacunas for hyperbolic equations, Mat. Sb., 17 (59) (1945), 289-370. 